\title{
Les protestants dans la Seigneurie de Nicolet
}

\section{Denis Fréchette}

Volume 52, 1985

URI : https://id.erudit.org/iderudit/1007003ar

DOI : https://doi.org/10.7202/1007003ar

Aller au sommaire du numéro

Éditeur(s)

Les Éditions Historia Ecclesiæ Catholicæ Canadensis Inc.

ISSN

0318-6172 (imprimé)

1927-7067 (numérique)

Découvrir la revue

Citer cet article

Fréchette, D. (1985). Les protestants dans la Seigneurie de Nicolet. Sessions d'étude - Société canadienne d'histoire de l'Église catholique, 52, 69-76.

https://doi.org/10.7202/1007003ar

\section{Résumé de l'article}

Cette communication rappelle l'existence d'une communauté protestante dans la Seigneurie de Nicolet. Après avoir établi la population protestante par l'entremise des recensements officiels et situé l'environnement social et culturel dans laquelle cette Église vivait, elle étudie les registres de Nicolet pour essayer de donner une vue globale du phénomène : 75 ans de présence protestante dans un milieu très majoritairement catholique. Un rappel de la présence de protestants au Séminaire de Nicolet termine cet exposé.
Tous droits réservés @ Les Éditions Historia Ecclesiæ Catholicæ Canadensis Inc., 1985
Ce document est protégé par la loi sur le droit d'auteur. L'utilisation des services d'Érudit (y compris la reproduction) est assujettie à sa politique d'utilisation que vous pouvez consulter en ligne.

https://apropos.erudit.org/fr/usagers/politique-dutilisation/ 


\title{
Les protestants dans la Seigneurie de Nicolet
}

\author{
Denis FrÉCHETTE, prêtre \\ Président de la Société d' histoire régionale de Nicolet \\ RÉSUMÉ
}

Cette communication rappelle l'existence d'une communauté protestante dans la Seigneurie de Nicolet. Après avoir établi la population protestante par l'entremise des recensements officiels et situé l' environnement social et culturel dans laquelle cette Église vivait, elle étudie les registres de Nicolet pour essayer de donner une vue globale du phénomène: 75 ans de présence protestante dans un milieu très majoritairement catholique. Un rappel de la présence de protestants au Séminaire de Nicolet termine cet exposé.

Depuis la concession de la seigneurie de Nicolet par acte du 29 octobre 1672 à Arnoult de Loubias (Laubia), capitaine d'une compagnie du régiment de Carignan-Salières cantonné à Trois-Rivières, jusqu'à l'ạbolition de la tenure seigneuriale en 1854 , cette seigneurie aura une vie mouvementée: procès, vente par shérif, démembrement et que sais-je encore. Un historien patient se chargera sans doute un jour de retrouver ce fil d'Ariane pour nous conduire sans faille au régime municipal établi sur le territoire de Nicolet le 31 juillet 1855 .

Notre propos se veut plus modeste et commence avec.la vente de la seigneurie par shérif à Kennelm Conor Chandler, le 6 mars 1821. Ce capi- 
taine du $60^{\mathrm{e}}$ régiment de l'armée anglaise, le Royal American, nouveau seigneur de Nicolet, est protestant et unilingue anglais. Dans un territoire presqu'exclusivement peuplé de Canadiens français catholiques, un double écueil surgissait: la langue et la religion. Le notaire Luc-Michel Cressé devient l'homme de confiance du nouveau seigneur et assure la communication avec les censitaires: l'écueil de la langue est aplani. Chandler entre bientôt en conflit avec le curé de Nicolet, l'abbé Jean Raimbault, et règle lui-même le problème de la religion... mais n'anticipons pas.

\section{Les recensements}

La cinquantaine de colons établis vers 1670-1672 par Pierre Mouet, sieur de Moras, et Arnoult de Loubias - d'après les actes de l'état civil et les greffes de notaires - ne forment plus que six ou sept familles lors du recensement nominal de Nicolet en $1681^{1}$. Il ne faudrait pas s'étonner outre mesure de cette baisse de population car, nous le savons, plusieurs préfèrent mendier après uñ essai infructueux de colonisation - phénomène de la «gueuserie» en Nouvelle-France - alors que le plus grand nombre cherche l'aventure et la subsistance dans la traite de fourrures. Cent ans plus tard, en 1781, les censitaires sont une centaine et lorsque l'arpenteur du Bas-Canada, Joseph Bouchette, fait le relevé de la seigneurie de Nicolet en 1815 , il dénombre « 250 concessions en très bon état de culture qui produisent de bonnes récoltes de grain de presque toutes les espèces» ${ }^{2}$.

Depuis le début du régime anglais, l'immigration de colons venus d'Angleterre et d'Écosse avait été favorisée et Londres s'était efforcé d'assurer le développement de l'Église anglicane dans sa nouvelle colonie par l'envoi de pasteurs auxquels de généreuses subventions étaient accordées. De plus, vers 1783-1784, 6,000 Loyalistes s'installent à la limite de la zone seigneuriale, dans les Townships, et forment le noyau initial d'une colonisation agricole anglaise dans la vallée du Saint-Laurent. Par suite de la migration des Loyalistes, les anglicans progressent rapidement et en 1789 Québec est érigé en évêché anglican.

Quand Chandler prend possession de sa seigneurie en 1821, y avait-il déjà des protestants censitaires? Sans doute, car la même année, le Lord Bishop of Quebec, le très révérend Jacob Mountain, nomme John Campbell Driscoll «pasteur de Rivière du Loup (Louiseville) avec charge de

\footnotetext{
${ }^{1}$ Elzéar Bellemare, ptre, Histoire de Nicolet 1669-1924, $l^{\text {re }}$ partie: La Seigneurie, Imp. d'Arthabaska, 1924, p. 49.

2 Joseph Bouchette, Topographie du Canada, Londres, 1815, p. 342.
} 
s'occuper des protestants de Machiche (Yamachiche) et de Nicolet ${ }^{3}$. La liste des souscripteurs ${ }^{4}$ pour la construction de l'église protestante en 1823-1824 contient seize noms de résidents dans la seigneurie et trois de Baie-du-Febvre. Cela nous donne une idée de la population protestante, en supposant que tous les chefs de famille aient souscrit soit environ quatrevingt personnes. Quelques années plus tard, en 1827, le pasteur Francis Evans donne le premier dénombrement de ses ouailles: «115 protestants à Nicolet dont 80 sont de l'Église anglicane» ${ }^{5}$.

Il nous faut attendre le recensement paroissial du curé Raimbault, en date du 15 octobre $1831^{6}$, pour recueillir enfin des détails fort intéressants sur la population protestante:

POPULATION: 3453

Catholiques: 3335 (96.6\%)

Non catholiques: $118(3.4 \%)$

Église d'Angleterre: 108

Presbytériens: 8

Église d'Écosse: 1

Autre dénomination: 1

Le métier ou la profession du chef de famille:

$\begin{array}{ll}\text { Seigneur: } 1 & \text { Constructeur de moulins: } 1 \\ \text { Médecin: } 1 & \text { Scieur: } 1 \\ \text { Notaire: } 1 & \text { Cultivateurs: } 8 \\ \text { Marchand: } 1 & \text { Journaliers: } 4 \\ \text { Militaire: } 1 & \text { Veuve: } 1 \\ \text { Ferblantier: } 1 & \end{array}$

Dans sept de ces vingt-et-une familles protestantes vivent treize catholiques et une seule famille catholique héberge un journalier sans dénomination religieuse connue.

Lors du premier «Recensement des Canadas» en 1851-52, donc vingt ans plus tard, la seigneurie ne compte plus que quatre-vingt-onze protestants dont cinquante sont de la paroisse de Sainte-Monique érigée en 1842. La population protestante ne cessera de diminuer si bien qu'au début du $\mathrm{XX}^{\mathrm{e}}$ siècle elle aura pratiquement disparue. Les Townships se développent rapidement, l'industrialisation commence, des routes s'ouvrent et

\footnotetext{
${ }^{3}$ Arthur E.E. Legge, The Anglican Church in Three Rivers, Quebec, 1768-1956, p. 147.

${ }^{4}$ Subscription List (copy), Archives du Séminaire de Nicolet (désormais ASN), L1,1,1.

${ }^{5}$ Legge, The Anglican Church, pp. 49-50.

${ }^{6}$ Recensement de la Paroisse de Nicolet, 15 octobre 1831, ASN, 6 bis.
} 
Nicolet qui comptait 3764 habitants en 1881 ne sera plus qu'un village de 1108 habitants.

\section{L'église Saint-Barthélémy}

La coutume d'offrir le pain bénit au seigneur, à sa femme et à ses enfants occupant le banc seigneurial faisait partie des privilèges établis par le régime seigneuriai ${ }^{7}$. Lors de ia fête paironaie célébrée le 4 novenııre 1821 , le curé Raimbault n'offre pas le pain bénit à la seigneuresse protestante qui prend place au banc seigneurial. C'est la seconde fois qu'un tel honneur lui est refusé. On avait cru à un oubli... cette fois Chandler demande $d \epsilon s$ explications, voire des réparations. Cette querelle du pain bénit $^{8}$ allait être portée devant les tribunaux quand finalement le seigneur se ravise, «met un peu d'eau dans son vin» et laisse tomber l'affaire.

S'il a perdu cette bataille, le seigneur protestant n'a pas perdu la guerre: une église de sa propre confession sera bâtie près de son manoir". En effet le 26 mai 1823 les contrats sont accordés et le 16 septembre de l'année suivante l'église Saint-Barthélémy est entièrement terminée et l'entrepreneur payé.

Dès que Jean Raimbault est mis au courant de cette construction, il s'inquiète du salut éternel de ses paroissiens engagés dans la construction d'une église hérétique. Après quelques admonestations, il avoue avoir relu saint Alphonse de Liguori sans avoir trouvé de réponses satisfaisantes au problème de la «coopération des ouvriers» dans une telle entreprise. Il cesse d'admonester ses ouailles et s'en ouvre à son évêque, Monseigneur Octave Plessis, qui lui conseille de consulter les moralistes de Montréal. L'église est achevée avant qu'une réponse définitive ne soit donnée.

Au sujet de la participation des catholiques aux enterrements des protestants, $\mathrm{M}^{\mathrm{gr}}$ Plessis n'hésite pas un instant à trancher la question que lui soumet son ami Raimbault: «les catholiques doivent s'éloigner de toute fonction, dans ces funérailles, qui les mettrait dans la nécessité d'accompagner le corps dans l'église ou dans le cimetière» ${ }^{10}$. S'il faut en croire le

\footnotetext{
7 Jugements du Conseil Supérieur de la Nouvelle-France, vol. V, 1705-1709, 8 juillet 1709, p. 999.

8 Denis Fréchette, «La querelle du pain bénit dans la Seigneurie de Nicolet», Les cahiers nicolétains, vol. 1, no 1 (février 1979), pp. 19-33.

${ }^{9}$ Denis Fréchette, «Une église protestante à Nicolet: Saint-Barthélémy», Les cahiers nicolétains, vol. $1 \mathrm{n}^{\circ} 3$ (octobre 1979), pp. 5-14.

${ }^{10}$ Lettre de $\mathrm{M}^{\mathrm{gr}}$ Plessis à Jean Raimbault, 11 novembre 1824, ASN, vol. 2 p. 442.
} 
registre protestant, cette directive est suivie à la lettre et aucune signature de catholiques n'apparaît sauf au bas des derniers actes consignés.

Au moment de sa démolition en novembre 1916, l'église de pierre est dans un piètre état; personne ne s'en était soucié depuis longtemps et le vandalisme avait hâté sa détérioration. Les quatre plaques commémoratives de la famille Chandler qui ornaient les murs sont aujourd'hui à l'église Saint James de Trois-Rivières et une croix de fer, aux archives du Séminaire de Nicolet. Ce sont les seuls vestiges de l'église du seigneur Chandler, les pierres ayant à leur tour disparu dans la démolition de l'École normale de Nicolet.

Les appréhensions du curé Raimbault au sujet d'une école anglaise à Nicolet alors que « $\mathrm{M}^{\mathrm{r}}$ Chandler et Co ont commencé à établir une petite maison pour une école qu'ils se proposent de tenir solidairement les dimanches»" ${ }^{11}$, sont-elles justifiées? L'abbé Bellemare affirme qu'une «école anglaise [est] tenue par $\mathrm{M}^{\text {lle }} \mathrm{Jane}$ Marler pour les jeunes anglais et quelques Canadiens français désireux d'apprendre l'anglais» ${ }^{12}$. Il ne cite pas ses sources et aucun document ne permet de corroborer son affirmation qui semble tout-à-fait plausible.

\section{Le registre protestant}

Le registre de l'église Saint-Barthélémy de Nicolet, aujourd'hui conservé aux archives de l'évêché anglican de Québec, est officiellement daté du 31 décembre 1827 et ne porte que la mention «Protestant Congregation of Nicolet» sans indiquer que c'est de l'Église anglicane qu'il s'agit, comme il appert tout au long de ce document. Ce registre comporte 176 folios dont à peine 85 contiennent les actes officiels des baptêmes, mariages et sépultures.

Notons que les trois premières inscriptions sont antérieures au 31 décembre 1827 et que les sept premiers folios (4 mai 1823-1 août 1827) sont des transcriptions des actes originaux du registre de Rivière-duLoup faites par le révérend Driscoll qui a charge des protestants de Nicolet et des environs depuis 1821 .

\footnotetext{
11 Jean Raimbault à $\mathrm{M}^{\mathrm{gr}}$ Plessis, 8 octobre 1825, ASN, vol. 2, p. 651.

12 Bellemare, Histoire de Nicolet, pp. 243-244.
} 


\section{Les baptêmes}

Le registre contient 245 baptêmes et 5 réceptions à l'Église d'Angleterre dont un méthodiste et quatre enfants déjà baptisés par le curé de Nicolet. Sans doute ces enfants de parents protestants avaient-ils été baptisés à l'Église catholique dans des cas d'urgente nécessité. D'ailleurs aucune conversion au protestantisme n'est notée dans le registre. Le prosélytisme que craignait tant le curé Raimbault ne porta pas fruit! À peine 6 baptêmes d'adultes encore qu'ils ne résident pas dans la seigneurie.

Il est intéressant de noter que les enfants nés de mariages mixtes sont, pour le premier, ordinairement baptisés à l'église protestante et que les suivants se retrouvent dans les registres de la paroisse catholique de Nicolet ou celle de Sainte-Monique. Ont été baptisés à Nicolet: le docteur Calvin Alexander, converti au catholicisme en 1842 et grand-père de $\mathrm{M}^{\mathrm{gr}}$ MoïseGeorges Proulx; les 11 enfants du pasteur Henry Burges; Jane Henrietta Robins, seconde épouse d'Ignace Gill, député d'Yamaska en 1854.

\section{Les mariages}

Des 63 mariages inscrits au registre, 19 le sont entre protestants et catholiques. Ces quelques mariages mixtes étaient inévitables dans un milieu relativement clos et à un moment où les déplacements étaient rares et difficiles. On peut penser que M. Raimbault ne voyait pas d'un bon oeil ses paroissiens ou paroissiennes s'unir à des personnes d'une autre religion, mais le coeur avait sans doute aussi ses raisons à cette époque...

À partir de 1856 et jusqu'à la désaffectation de l'église protestante, à peine 6 mariages furent célébrés. La communauté anglicane de Nicolet, si le nombre de mariages est un indicateur de développement et de vitalité, aura eu quelque 25 ans de prospérité. Se sont mariés à Nicolet: le notaire Luc-Michel Cressé et Margaret Van Cortland; Pierre-Nérée Dorion et Mary Ann Marler; Leonard Marler et la seigneuresse Mary Ann Collins Woodward; les deux petites filles du seigneur, Henrietta et Jane Elizabeth Trigge.

\section{Les sépultures}

Le registre contient 93 actes de sépultures du 13 juin 1824 au 29 mai 1908. Le 16 septembre 1824, pour la première fois, il est fait mention d'un enterrement dans le cimetière protestant de Nicolet. À partir de 1873 les 
inhumations se font ordinairement en dehors même si le service religieux se tient à Nicolet. D'après les registres de l'Hôtel-Dieu de Nicolet, le 19 mai 1942 une dernière sépulture eut lieu au cimetière protestant: William Boleyn, pensionnaire chez les soeurs Grises, décédé à l'âge de 93 ans.

Le tableau ci-dessous semble confirmer les statistiques que nous connaissons de la forte mortalité infantile au $\mathrm{XIX}^{\mathrm{e}}$ siècle:

$\begin{array}{rl}0 \text { à } 5 \text { ans: } 34 & 21 \text { à } 30 \text { ans: } 4 \\ 5 \text { à } 14 \text { ans: } 5 & 30 \text { à } 60 \text { ans: } 19 \\ 14 \text { à } 18 \text { ans: } 3 & 60 \text { et plus: } 14 \\ 18 \text { à } 21 \text { ans: } 2 & \text { sans âge: } 12\end{array}$

Aujourd'hui le cimetière anglican, restauré par les soins de l'évêque de Nicolet et de la Société d'histoire, est entretenu aux frais de la paroissecathédrale. Une dizaine de pierres tombales et le caveau du seigneur Chandler qui était sous l'église, rappellent humblement le passage des protestants dans l'histoire de la seigneurie de Nicolet au siècle dernier. Sont inhumés à Nicolet: le seigneur Chandler; son gendre, Thomas Trigge; les marchands de Baie-du-Febvre, William et Murdoch MacKenzie. L'apostat Georges-Jérémie Pacaud y eut son service religieux.

\section{Les protestants et le séminaire}

Le Séminaire de Nicolet accueille très tôt des étudiants de religion protestante. Dès 1821 trois jeunes y sont admis: Adolphus Alexander, William et Francis Gilmor. Quelques années plus tard on relève les noms de deux petits-fils du seigneur Chandler, Alfred et Henry-Wulff Trigge ainsi que les fils de ministres protestants desservant Nicolet: Henry Burges et Joseph de Mouilpied.

Il est difficile d'établir le nombre exact d'étudiants non-catholiques ayant fréquenté cette institution. Le seul rapport officiel ${ }^{13}$ retrouvé donne pour les années 1859 à 1880 le nombre de 82 protestants sans jamais dépasser 8 étudiants pour une seule année; entre 1821 et 1859 , on en compte une quarantaine. Comme il faut tenir compte du fait que plusieurs reviennent plus d'une année, on peut dire qu'environ 75 jeunes protestants, dont une cinquantaine de Nicolet et des environs, ont été étudiants au Séminaire de Nicolet.

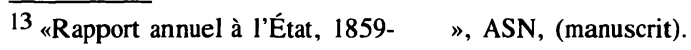


En 1842 , le règlement du séminaire dicté par l'évêque de Québec, $\mathrm{M}^{\mathrm{gr}}$ Joseph Signay, consacre un article à la bonne entente entre catholiques et protestants:

Il est strictement défendu durant les récréations, comme en tout autre temps que ce soit, de faire aucun reproche, aucune réflexion, ou tenir aucun propos qui tend à railler les croyances ou les pratiques religieuses de ceux des élèves qui ne sont pas catholiques. Cette défense regarde pareillement ces derniers à l'égard de leurs compagnons catholiques. ${ }^{14}$

Ajoutons que tous les étudiants sont obligés d'assister à tous les exercices de la maison et de se tenir avec respect à la chapelle et ailleurs. Nous pouvons nous demander ce que valait cette participation! Quoi qu'il en soit, elle n'a pas semblé peser trop lourd sur ces jeunes écoliers comme en témoignera plus tard l'avocat Edward Carter, au conventum du 24 mai 1866:

Aussi je me plais à le déclarer en face de cette illustre assemblée, jamais je n'ai eu dans tout le cours de mes classes, la moindre occasion de souffrir dans mes croyances religieuses. [...] Ce souvenir est impérissable car c'est ici que j'ai appris à respecter votre clergé. ${ }^{15}$

C'est avec ce témoignage non-équivoque que je termine cet exposé. Au delà de quelques stèles funéraires qui se dressent encore dans le cimetière anglican de Nicolet, n'oublions pas que ces hommes et ces femmes qui ne partageaient pas notre foi participaient avec la même détermination que la nôtre à l'établissement de notre coin de patrie.

14 «Règlement du Séminaire de Nicolet, 1 juin 1842», ASN, Sém. I, nº 37 bis, (manuscrit).

${ }^{15}$ Le Séminaire de Nicolet, "Conventum du 24 mai 1866», Montréal, La Minerve, 1867, p. 102. 